\title{
INTERVIEW WITH SANDEEP GAUTAM, MD, FHRS
}

\section{ANGELINA WAGNER}

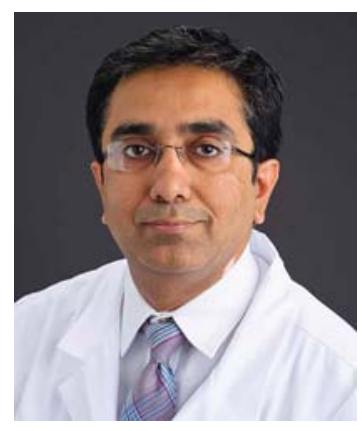

\section{Introduction}

Radiation exposure during ablation for cardiac arrhythmias has long been considered a "necessary evil," with the potential of radiation exposure during cardiac ablation procedures well documented. Despite the improvements in electroanatomic mapping techniques over the last 20 years, fluoroscopy continues to be the primary imaging modality for intracardiac ablation procedures in the adult patient, although fluoroless ablation is being practiced in the pediatric population. The ALARA (as low as reasonably achievable) principle maintains that there is no measurable lower safe dose for radiation, and encourages "every reasonable effort to maintain exposures to ionizing radiation as far below the dose limits as practical." There have been some reports and case series of both simple and complex arrhythmias in adults being performed without fluoroscopy, mostly using a combination of three-dimensional electroanatomic mapping techniques, intracardiac echocardiogram (ICE), and remote magnetic navigation systems. However, use of fluoroless ablation remains extremely sporadic, most likely owing to concerns about procedural safety, efficacy, and efficiency.

Dr. Sandeep Gautam, MD, FHRS is Assistant Professor of Clinical Medicine, Division of Cardiovascular Medicine at the University of Missouri, Columbia, MO. The results of his study evaluating fluoroless radiofrequency ablation (RFA) of typical cavotricuspid isthmus-dependent atrial flutter have demonstrated that the procedure can be safely and efficiently performed without the use of fluoroscopy. This finding has important implications for the reduction of radiation exposure to patients as well as operators and staff during ablation.

We speak with Dr. Gautam about the clinical research in fluoroscopy-free procedures, and his perspectives and discernments on how to achieve fluoroscopy free procedures.

Wagner: In your research, what changes have you seen in patient care to reduce the risks of radiation exposure?

Gautam: The most important change has been an increasing willingness on the part of physicians (electrophysiologists) to acknowledge the dangers posed by radiation, both to the patient and the operator. For too long, the EP/cath lab environment was dominated by the "step on the pedal" approach towards fluoroscopy. This change was initially driven by the development of better and lighter lead aprons and ceiling-mounted zero-gravity lead shields, and later by radiation-reducing techniques such as low fluoroscopic equipment, rotational angiography, and electroanatomic mapping.

Wagner: Tell us about your retrospective study "Fluoroless radiofrequency ablation of typical cavotricuspid isthmusdependent atrial flutter", 1 and the role that this procedural approach will have in clinical practice?

Gautam: In this study, we concentrated on the safety and efficiency components of fluoroless ablation for a simple arrhythmia such as typical right atrial cavotricuspid isthmus-dependent flutter. This procedure has been performed without fluoroscopy by a limited minority of electrophysiologists. In our retrospective study, we compared the outcomes of standard flutter ablation procedure in 20 patients with a similar number of patients who underwent completely fluoroless ablation guided only by electroanatomic mapping. We found that all procedural parameters including success, duration, and safety were identical between the two groups. Also, there was no significant difference in the procedural parameters between the first and second 10 patients in the fluoroless ablation groups, indicating a sharp learning curve. In short, fluoroless flutter was performed safely with similar procedural results, without compromising of lab efficiency.

Wagner: Do you foresee any limitations in the adoption of this approach? If so, how can these challenges be overcome?

Gautam: I sincerely believe that most, if not all, electrophysiologic catheter ablation procedures can be performed without fluoroscopy. The use of ICE enables adequate visualization of the intreratrial septum and intracardiac hardware to enable fluoroless transseptal access for left atrial ablation, as has been described by multiple authors. ICE mapping using the CARTOMERGE ${ }^{\mathrm{TM}}$ software (Biosense Webster, Inc., Diamond Bar, CA) can be used to create atrial or ventricular shells and delineate papillary muscles/moderator bands, etc., to allow fluoroless ventricular ablation procedures.

Potential limitations include ablation procedures in patients with new intracardiac electronic devices (pacemakers and defibrillators) placed less than a few months ago with potential of lead dislodgment. Patients with older devices can safely be ablated without fluoroscopy. 
Another limitation is the potential need for fluoroscopy in urgent situations like suspected pericardial tamponade. However, this can be easily addressed, as it hardly takes less than a minute for an experienced operator to lead up for emergent fluoroscopy. Problems with femoral/jugular venous access can be addressed by use of vascular ultrasound.

Wagner: Are there long-term safety concerns about the usage of fluoroscopy during cardiac ablation procedures?

Gautam: According to the ALARA principle, there is no measurable lower safe dose for radiation. The ACC Expert consensus document in 1998 on radiation safety in the practice of cardiology details the possible deterministic (dose-related) and stochastic (dose-independent) effects of radiation, including erythema, desquamation, cataracts, decreased white blood count, organ atrophy, fibrosis, sterility, cancers, and genetic birth defects in offspring. These apply both to the patient and the operator. In addition, of course, we have to consider the long-term concern for chronic back pain in the operator and EP lab personnel due to lead aprons, which are completely avoided in fluoroless procedures.

Wagner: What alternatives to using fluoroscopy during cardiac ablation procedures are available?

Gautam: Perhaps the most important progress in electrophysiology has been the remarkable development in the field of electroanatomic mapping, with the resultant ability to create exact cardiac anatomical maps and visualize all intracardiac catheters. ICE, as mentioned above, is the other technological innovation that has enabled us to safely perform fluoroless complex ablations.

Wagner: How has electroanatomical mapping optimized patient outcomes to achieve fluoroscopy-free procedures?

Gautam: Electroanatomic mapping, offered by multiple vendors, utilizes a combination of impedance and magnetic field mapping to create three-dimensional representational maps, which can be used to supplement or replace fluoroscopy. Nearly all vendors now have the capacity to allow fluoroless visualization of intracardiac catheters, and to allow catheter visualization in the iliac vasculature and IVC/aorta to enable catheter advancement and placement. In addition, electroanatomic mapping can be used to visualize virtual biplane imaging, and indeed obtain multiple views at various orientations, which is actually an advantage over traditional fluoroscopy in most EP labs.

Wagner: How does the comparison between standard fluoroscopy-guided ablation and fluoroless ablation demonstrate procedural feasibility?

Gautam: As demonstrated in our flutter ablation study, fluoroless ablation demonstrated excellent procedural feasibility. ${ }^{1}$ In the same issue of the Journal of Innovations in Cardiac Rhythm Management, Montgomery \& Ellis published a case series of 14 fluoroless atrial fibrillation ablation procedures. ${ }^{2}$ In our experience, fluoroless ablation can be performed easily and efficiently, and is much preferred by the EP lab staff.

Wagner: What technical procedural skills should physicians have for these procedures?

Gautam: I strongly believe that any experienced electrophysiologist can easily and rapidly develop the required procedural skills for fluoroless ablation, as demonstrated in our study with the finding of similar procedural parameters in the first and second 10 patients undergoing fluoroless flutter ablation. In our experience, the learning curve here is quite sharp, and mainly requires the operator to let go of the apprehension of not "seeing" the catheters.

Wagner: How do specific protocols for patient assessment and care play a key factor into achieving fluoroscopy-free procedures?

Gautam: Radiation safety is an important patient care assessment parameter, with requirements for patient skin assessment, usually for procedures with fluoroscopic exposure longer than 60 minutes. Interestingly, there does not appear to be any clear system to monitor radiation exposure to patients from multiple imaging studies, etc., in addition to coronary angiography, interventional radiologic procedures, and EP ablation.

Wagner: What advancements in fluoroscopy-free procedures do you see on the horizon?

Gautam: I expect fluoroless ablation procedures to be the norm down the road. I expect further developments to allow transseptal access under electroanatomic mapping guidance, without the need for ICE. Already, there are trials on transseptal needles which can be integrated into the mapping system. I would encourage physician workshops and training seminars to work towards increasing comfort with fluoroless procedures. Further refinements in electroanatomic mapping, including contact force sensing, rapid activation mapping, and extremely high-density mapping, will make the procedures shorter and more efficient.

Wagner: Could you please speak to the importance of further studies, such as the ongoing NO-PARTY trial, surrounding measuring the cost-effectiveness of the RFA for typical atrial flutter approach, and its utility in ablation of other cardiac arrhythmias?

Gautam: One of the oft-repeated arguments against fluoroless ablation is the requirement for electroanatomic mapping, with increased procedural cost. I agree that simple arrhythmias like typical AVNRT and CTI-dependent atrial flutter can be safely ablated using only fluoroscopy. However, this approach is rapidly disappearing, with the vast majority of ablation procedures nowadays utilizing electroanatomic mapping. 
The NO-PARTY trial is a planned randomized controlled trial which aims to determine whether radiofrequency catheter ablation of supraventricular tachycardias using the EnSite NavX ${ }^{\mathrm{TM}}$ mapping (St. Jude Medical, St. Paul, MN) system is a suitable and cost-effective approach to achieve a clinically significant reduction in ionizing radiation exposure for both patient and operator. This will be a multicenter trial, utilizing multiple operators, with anticipated randomization of 210 patients. This will be an advantage over published care series and small-scale RCTs, which have mainly been reported by single or limited operators. The trial will also look at cost-effectiveness as a secondary endpoint. It will also include evaluation of radiobiological effectiveness of dose reduction by assessing acute chromosomal DNA damage in peripheral blood lymphocytes in a subgroup of patients.

Wagner: What improvements need to be made to speed access to minimizing and ultimately eliminating the use of fluoroscopy from most cardiac ablation procedures?

Gautam: As I have mentioned above, I would encourage industry and physician organizations (such as HRS and EHRA) to organize physician workshops and training seminars designed to increase comfort with fluoroless procedures. As responsible physicians, we need to spread the word and educate fellow professionals about the dangers of radiation and ALARA concept, and the available modalities to reduce and eliminate fluoroscopy from catheter-based ablation procedures. Ultimately, this needs to be engrained during training of electrophysiology fellows.

\section{References}

1. Gautam S, Jain R, Miller JM. Fluoroless radiofrequency ablation of typical cavotricuspid isthmus-dependent atrial flutter. J Innov Cardiac Rhythm Manage. 2015;6(12):2201-2207.

2. Montgomery J, Ellis C. Zero-fluoroscopy Intracardiac Echocardiography-guided Ablation of Atrial Fibrillation Using a Singlecatheter Technique. J Innov Cardiac Rhythm Manage. 2015;6(12):2209-2215. 